\title{
Germanica
}

\section{Réécrire les contes de Grimm aujourd'hui}

Grimms Märchen, wie sie heute umgeschrieben werden

\section{Christian Klein}

\section{(2) OpenEdition}

\section{Journals}

Édition électronique

URL : http://journals.openedition.org/germanica/1334

DOI : 10.4000/germanica.1334

ISSN : 2107-0784

Éditeur

Université de Lille

\section{Édition imprimée}

Date de publication : 1 janvier 1992

Pagination : 19-42

ISSN : 0984-2632

Référence électronique

Christian Klein, « Réécrire les contes de Grimm aujourd'hui », Germanica [En ligne], 11 | 1992, mis en ligne le 07 février 2014, consulté le 06 octobre 2020. URL : http://journals.openedition.org/germanica/ 1334 ; DOI : https://doi.org/10.4000/germanica.1334

Ce document a été généré automatiquement le 6 octobre 2020.

(C) Tous droits réservés 


\title{
Réécrire les contes de Grimm aujourd'hui
}

\author{
Grimms Märchen, wie sie heute umgeschrieben werden
}

\author{
Christian Klein
}

1 Cette étude présente quelques réflexions qui nous ont été inspirées par un atelier d'écriture de quatre semaines animé à la Pädagogische Hochschule de Ludwigsburg en collaboration avec Monsieur Hartmut Melenk, professeur de linguistique, dans le cadre d'un échange d'enseignants avec l'Université Stendhal de Grenoble ${ }^{1}$. Les étudiants travaillent dans leur langue et culture maternelles. Ils devaient réécrire des contes de Grimm et tenter d'étudier, à partir de ces exercices, des structures fondamentales du conte. Nous nous sommes plus particulièrement attachés aux objectifs suivants :

2 - dégager, à travers les variantes, un canevas et des constantes qui permettent de retrouver les fonctions définies par Propp et reformulées par Claude Brémond dans son schéma triadique (virtualité, actualisation, résultat ${ }^{2}$ ). Nous pouvons distinguer des déplacements dans l'espace, un manque ou un méfait initial, des épreuves (initiale, centrale, finale) du héros. Soit, dans le cadre de notre propos: 1) éloignement ou absence (mort de la mère, départ des parents), 2) interdiction (ou forme inverse de l'ordre), 3) transgression, etc...

3 Au-delà de cette analyse, qui porte sur la succession des séquences, nous avons recherché d'autres aspects :

les modifications du schéma initial bon/mauvais.

- les actualisations au niveau du vocabulaire, du contexte socio-culturel.

- la politisation de la fable.

- l'investissement personnel de l'étudiant(e), qui substitue son expérience personnelle, ses phantasmes, aux thèmes narratifs de référence.

- le plaisir de produire un texte à partir d'un modèle, tantôt de le copier par un réflexe de mimétisme, tantôt de le parodier ou de le déconstruire. Chaque production a été lue en séance et a fait ensuite l'objet d'un inventaire collectif de ce qui variait, tant au niveau de la structure que de l'écriture (langage, références intertextuelles). 


\section{«Rotkäppchen»}

Une première série traite du conte « Le petit chaperon rouge » de Grimm.

Document 1

\section{Rothärchen und der Macho}

Rothärchen legt den Hörer auf. Eben hat sie mit ihrer älteren Freundin telefoniert und sich für einen Besuch verabredet. Diese, einst eine engagierte, temperamentvolle Frau, liegt seit Wochen depressiv und gefrüstet im Bett und jammert irgend so einem Kerl nach, der sie in typischer Machoart verlassen hat... Was ist nur aus Rosenweißchen geworden!? Eine intelligente, selbstbewußte Frau, die sich so gehen läßt. Es ist nicht zu glauben. So was kann mir nicht passieren!... Sie stellt sich vor den Spiegel und betrachtet sich: ... «Eigentlich sehe ich ganz gut aus», sagt sie zu ihrem Spiegel. Mit großer Hingabe legt sie noch etwas Rouge auf, die Lippen werden in kokettem Rot nachgezogen und die Haare in Vampmanier auftoupiert. In engem Minirock, hochhackigen Schuhen und Lederjacke schwingt sie sich in ihr knallrotes Capriolet und Starter in Richtung zur Freundin. An der Ausfahrt «Waldesruh» steht ein Tramper. Als selbstbewußte, starke Frau hat sie keine Scheu, den dunkelgelockten, verwegen aussehenden Mann mitzunehmen...! Uhu, was hat er nur für wunderschöne Augen und süße Öhrchen. Auch der Mund ist geradezu zum Knutschen geschaffen...!, geht ihr durch den Sinn, fast wie im Märchen!... der Mann bietet ihr eine Zigarette an, die sie bereitwillig annimmt. «Mein Auto ist gerade in Reparatur. Nicht, daß Sie denken, ich sei so eine dahergelaufene Gestalt, die sich auf Kosten von anderen durchs Leben schlägt»... Sie rauchen eine Weile schweigend. Die elektrisierende Spannung wächst. Rothärchen malt sich in Gedanken schon in den buntesten Farben die Fortsetzung dieser Begegnung aus. «Übrigens, ich heiße Haribert Wolf!» Rothärchen tritt auf die Bremse, öffnet die Beifahrertür und sagt: «Bitte steigen Sie aus. Wir sind da». Irritiert verläßt der Mann den Wagen, der sofort wieder durchstarter. «Gerade davongekommen», denkt Rothärchen und fährt weiter.

Commentaires des participants :

- la fin a été changée : prévenue par les péripéties du conte, le chaperon rouge d'aujourd'hui évite les erreurs du modèle ;

- on relève des emprunts à d'autres contes de Grimm³ ;

- le topos de la beauté a changé : les femmes doivent (veulent) être belles et soignent leur visage, leur corps. Dans le conte, la beauté est une donnée acquise et abstraite ;

- inversion de l'opération de séduction : la jeune femme n'est plus victime mais agent ;

- le lecteur est surpris dans son horizon d'attente ;

- l'écriture recourt : a) au vocabulaire contemporain, familier ; b) au monologue intérieur ;

c) à des citations empruntées au patrimoine commun des contes populaires.

On note l'apparition d'un métalangage («fast wie im Märchen»), dont la fonction indicielle est de signaler ici la règle de l'exercice et de cautionner la liberté - d'un type particulier qu'il reste à définir - que s'autorise l'auteur.

- D'autres éléments : 
7 a) La phase préliminaire du récit (le mini-récit antérieur) sert à la fois d'exposition référentielle et de contre-modèle qui génère, par opposition, une modification du récit initial.

b) L'instance narrative intervient dans le récit par une série de commentaires («als selbstbewußte, starke Frau hat sie keine Scheu...»). On surprend ici une (con)fusion entre l'instance narrative et le personnage.

c) La narration est dominée par une certaine ambivalence («fast wie im Märchen») : on est et on n'est pas dans un conte. D'où des libertés nouvelles, comparables à la permissivité du rêve, qui fait jouer le procédé de la dénégation («Verneinung») par lequel le sujet formule un désir tout en niant qu'il lui appartienne. L'activité littéraire se donne à lire comme un jeu qui masque des enjeux plus sérieux.

8 L'étudiante affirme un désir d'émancipation qui est lié à son parcours de formation encore inachevé et, plus ou moins consciemment, au mouvement féministe allemand des années soixante-dix. Le loup est devenu un macho, le chaperon n'est plus un être naïf et sans défense, mais une personnalité consciente de ses atouts. D'autres versions imaginent qu'elle simule une panne pour séduire son voyageur etc. La volonté d'émancipation se confirme avec l'absence de la grand-mère et de toute instance parentale. Il n'y a plus de recours à des adjuvants, comme le chasseur dans le conte de Grimm, pour sauver une situation compromise.

9 D'autres variantes tentent de transposer un canevas plus conforme au modèle: le chaperon est une victime innocente (une juive, une jeune fille très aimée dans son village ou son quartier), le loup est alors un nazi, ou un sadique. Le chasseur est américain ou un voisin, un représentant de l'ordre.

On peut chercher ce qu'il reste encore de la structure initiale du conte. La mission, le déplacement hors de chez soi («Heim», «Wohnung») vers un lieu familier (une amie, une parente). La forêt, lieu de tous les dangers, devient une autoroute, symbole moderne de l'évasion et de l'aventure. L'innocence du sujet, qui incarne le bon droit ${ }^{4}$. Dans le document 1 , le personnage n'est plus innocent, mais occupe la place de la « victime » traditionnelle qui « dialogue » avec son modèle et entend bien modifier le cours des événements. Cependant, les présupposés culturels et idéologiques sont non seulement différents et actualisés, mais ils se définissent par rapport aux valeurs du récit de référence dont ils affirment la contestation. L'identité du méchant est liée à la notion de couple (femme émancipée/macho, juive/nazi, turque/raciste, etc.). L'axe paradigmatique ouvre des séries illimitées.

11 À partir d'un tel schéma transposé, il reste à inventer un récit et en particulier les dialogues. L'appel à la productivité langagière est toujours lié au plaisir d'inventer une parole, de réutiliser des expressions familières à l'enfant ou à l'étudiant (en langue étrangère comme en langue maternelle) et souvent exclues du discours scolaire ou universitaire. La maîtrise du verbe ne passe alors plus par assimilation mimétique d'un discours codé (celui de l'analyse littéraire), mais par une appropriation vivante et narquoise de stéréotypes empruntés aux média, ou à l'oralité. L'identification de l'étudiante avec son personnage fictif, dans le document 1 , donne lieu à un engagement personnel par récit interposé et modifie en conséquence le rapport entre enseignant et enseigné. 
Il importe ici de distinguer transposition et réécriture. La transposition d'un texte canonique se limite à un exercice intellectuel qui met en jeu une certaine virtuosité. Prenons l'exemple suivant:

Document 2 :

\section{Rotkäppchen auf Reklamedeutsch}

Es war einmal ein Kind, das hieß Rotkäppchen, weil es kein Käppchenmuffel war, sondern stets ein hautverträgliches Hütchen trug, das war röter als das röteste Rot unseres Lebens, dazu kochecht, absolut waschmaschinenfest, mit doppeltem Mittelstück und patentiertem Verschluß. Zu dem sprach die Mutter, eine nicht alltägliche Frau, da Skunksin ihr einen reinen Atem verleiht: «Mach mal Pause, pack den Tiger in den Tank und geh meilenweit zur rieselfreudigen Großmutter, denn sie ist krank, weil sie nicht bei der Mesallina Sach und Leben versichert ist. etc. ${ }^{5}$

Ce document est un exercice de style. Il peut se pratiquer comme un jeu récréatif, après un travail en atelier sur le langage publicitaire. On remarque :

a) que le schéma est rigoureusement respecté,

b) que l'intérêt de ce texte est déplacé complètement. Le récit initial est supposé connu. Tout le travail et l'intérêt sont concentrés dans la transposition : l'axe syntagmatique et la logique du récit sont conservés, le travail porte exclusivement sur l'axe paradigmatique, par accumulation d'extensions à droite, ou, pour d'autres versions, par substitutions lexicales,

c) cette technique de parasitage (ou, ailleurs, de transposition terme à terme) fait appel à une grande maîtrise des codes et de la langue, à la disposition d'importantes réserves en matériaux verbaux,

d) il n'y a pas d'investissement du sujet dans ce type de réécriture.

\section{« Jeannot et Margot »}

Une autre série de textes porte sur le conte de « Jeannot et Margot» («Hänsel und Gretel»).

Document 3 :

\section{Hänsel et Margerete}

Es waren einmal Bruder und Schwester, Hänsel und Margarete. Die lebten überhaupt nicht glücklich und überhaupt nicht zufrieden. Die böse Stiefmutter schien die Gestalt von Vater und Mutter anzunehmen. Doch die Eltern wollten nur ihr Bestes. Sie liebten ihre Kinder. Tu dies nicht, tu das nicht. Geh aufrecht. Ein Junge weint nicht. Das sind keine Ausdrücke für ein Mädchen. Ein Junge spielt nicht mit Puppen. Ein Mädchen zieht keine Hosen an. Jungen spielen Fußball. Mädchen lieben die Hausarbeit. Sie sind zurückhaltend, liebenswürdig, weiblich. Hänsel dagegen sind stark, mutig, fürchten keine Gefahr. Hänsel beschützen die Gretein. So ist das auch mit Vater und Mutter. Vater geht arbeiten, bringt den Unterhalt für die Familie auf, hat deshalb auch das Recht, alle Entscheidungen selbst zu treffen. Mutter erzieht die Kinder, versorgt den Haushalt und liebt Vater. 
Früh am nächsten Morgen macht man sich auf, leicht bekleidet - es ist Sommer mit einem Rucksack auf dem Rücken und etwas Geld in der Tasche. Wohin? Der naheliegende Wald ist auf. Von Ginster keine Rede. Leise rieseln die Nadeln. Der Wald ist krank.

Also erstmal nur Autobahn. Alles weitere wird sich finden. Da stehen sie nun, die beiden. Margarete hält den Daumen 'raus. Schließlich werden Mädchen bevorzugt mitgenommen. Und tatsächlich. Schon nach kurzer Zeit hält mit quietschenden Reifen und kreischenden Bremsen ein buntbemaltes, großbauschiges Automobil wenige Zentimenter vor Margaretes Nase an.

«Wohin wollt ihr denn?»

«Ziel unbekannt - vielleicht in ein anderes Land?» meint die Schwester ungeniert und etwas holperig.

«Ach nein - so steigt denn mal ein» kommt die Antwort schnell zurück. Fahrer und Beifahrer haben Humor.

Wie geht's weiter?

Nunja, wie das ebenso ist in einem richtigen Märchen. Unglaublich geht's weiter. Doch ist die Hexe nicht böse, will die Kinder nicht braten und verspeisen. Ganz im Gegenteil, sie ist liebenswürdig und hilfsbereit. In besagtem Automobil saßen nämlich zwei Andersdenkende. Leute, die ihr Leben zumindest versuchen, wenigstens ein bißchen anders zu gestalten als Normalbürger. Keine Eremiten nein; sie lebten mitten in der Stadt mit ein paar anderen zusammen, keine Eigenbrötler - doch so wie sie wollen. Sie lassen Hans Hans sein und Margarete Margarete. Sie wollen keine Hänsel und Gretchen sein.

- une triple rupture s'installe dès les préliminaires :1) «die lebten überhaupt nicht glücklich und überhaupt nicht zufrieden». Le nouveau récit donne - sous forme quasi citationnelle - la fonction initiale «manque » des contes populaires. Cette première séquence introduit déjà subrepticement le point de vue de l'enfant et annonce la mise en place d'un second schéma narratif. 2) «"die böse Stiefmutter" schien die Gestalt von Vater und Mutter anzunehmen». Le narrateur affiche une distanciation et impose sa présence en présentant le récit comme le produit d'une réécriture. Il met en perspective le récit premier. Dans le même temps, l'auteur se réfère à une structure qui détermine un jeu de rôles : le rôle de la marâtre (le "vilain ») étant assumé par le couple parental. On distingue bien un rôle et celui (celle, ceux) qui sont chargés de 
l'assumer. Le schéma actanciel devient ainsi plus lisible. 3) «doch die wollten nur ihr bestes». On assiste à la greffe d'un second schéma narratif sur le premier : le récit d'une émancipation de deux enfants contre une éducation autoritaire. Ce nouveau schéma narratif introduit le réfèrent socio-culturel de la narratrice: la R.F.A. des années soixante-dix et quatre-vingt, mais aussi le contexte particulier de production du récit. La Pädagogische Hochschule (modèle réussi de nos I.U.F.M.) accueille les jeunes gens, essentiellement des jeunes filles, issus des campagnes - et non des villes - du Wurtemberg. Ainsi s'explique, par exemple, le curieux rapport ambivalent à l'instance parentale: ils ont le rôle de la marâtre, mais ne sont pas vraiment méchants. Cette ambivalence traduit le compromis personnel de la narratrice avec la représentation parentale traditionnelle qui est le prix à payer pour exercer le droit à l'émancipation.

- la réécriture donne lieu à une réinterprétation et une redistribution des rôles. C'est ainsi que le couple actif/passif de Jeannot et de Margot devient un couple passif/actif qui revalorise le personnage de la fille.

Le travail en atelier d'écriture permet à de jeunes lecteurs / scripteurs de réinvestir un conte ancien à partir d'éléments empruntés à leur propre biographie. C'est ainsi que l'on retrouve des thèmes d'actualité (le milieu alternatif, la forêt malade) et que la sorcière du conte de Grimm change de statut. Chez les frères Grimm, les enfants rencontraient une vieille femme qui vit seule dans la forêt :

Die Alte hatte sich nur so freundlich angestellt, sie war aber eine böse Hexe, die den Kindern auflauerte, und hatte das Brothäuslein bloß gebaut, um sie herbeizulocken. Wenn eins in ihre Gewalt kam, so machte sie es tot, kochte es und aß es, und das war ihr ein Festtag6.

Dans le nouveau récit, la situation est très différente :

Doch ist die Hexe nicht böse, will die Kinder nicht braten und verspeisen. Ganz im Gegenteil, sie ist liebenswürdig und hilfsbereit.

Mais cette différence joue sur une ambivalence. En effet, Marthe Robert note que dans les contes allemands le personnage de la vieille femme "ne laisse pas d'être équivoque ", tantôt " puissance tutélaire », tantôt « sorcière acharnée au mal ». Qu'elle soit magicienne ou sorcière, elle préside, comme les Moires grecques, au destin de l'homme ${ }^{7}$. Sa rencontre est une épreuve et le conte décrit «un passage nécessaire, difficile, gêné par mille obstacles [...] mais qui s'accomplit heureusement à la fin en dépit de tout $"^{8}$. L'inversion aujourd'hui en signe plus du signe moins, qui affectait la " sorcière » dans l'édition de 1812, respecte donc la structure initiale en levant, certes différemment, l'ambiguïté du personnage central des contes de Grimm et en lui confiant le rôle de la "sage-femme " ${ }^{9}$, dépositaire des rites, initiatrice et conseillère. Elle est la personne sage dont les conseils aident l'enfant égaré du conte à sortir de son errance et à passer d'un état à l'autre, d'un âge à l'autre. À ce titre, la réécriture moderne des contes s'adresse en priorité à des individus en formation.

L'étudiant(e) affirme son désir d'autonomie par rapport à l'univers littéraire des contes de Grimm, par rapport aux livres de son enfance et à l'atmosphère familiale qui présidait à ces lectures ${ }^{10}$. Tout en prenant congé de ces années de formation, l'étudiant(e) découvre les bifurcations narratives mises en évidence par Claude Brémon ${ }^{11}$.

On notera enfin la pureté narrative du texte, qui reprend les trois moments de la fameuse triade de Brémond. Tout récit se développe selon trois moments (initial, médian, terminal) : 
29 1) Virtualité : fonction qui ouvre ou non la possibilité d'un processus [les enfants sont malheureux chez leurs parents].

2) Actualisation: fonction qui réalise ou non cette virtualité [les enfants quittent la maison familiale].

3) Résultat: fonction qui clôt le processus [les enfants sont accueillis dans une famille alternative].

On débouche alors sur un schéma par enclaves :

Chez Grimm on a :

32 1. l'abandon des enfants par les parents.

2. l'errance dans la forêt

a] dégradation potentielle.

b] processus de dégradation.

b1 amélioration à obtenir.

b2 processus d'amélioration.

b3 amélioration obtenue

c] dégradation évitée ${ }^{12}$.

Dans le nouveau texte on a :

33 1. le départ des enfants.

2. l'errance :

a] dégradation potentielle (attendue)

b] o

c] pas de dégradation à surmonter.

3. l'émancipation est réussie.

Ce schéma fait apparaître que le document 3 a évité les possibles narratifs : il suffit d'un déplacement géographique pour résorber le conflit. En d'autres termes, l'épreuve consiste à quitter ses parents. On découvre alors une réduction du schéma narratif, qui peut donner lieu à discussion, ou à des exercices qui consistent à réinventer des possibles refoulés.

$\mathrm{Au}$ cours de ce séminaire est apparu une limite: la substitution systématisée d'un schéma anti-autoritaire ou féministe à un schéma traditionnel conduit à deux cas de figure. Dans la mesure où cette substitution s'accompagne de modifications structurelles, il y a production verbale et réflexion sur la structure du conte (et du récit littéraire en général). Dans le cas contraire, il y a reproduction transposée d'un schéma préétabli, sans distance critique de la part de l'étudiant ni sur l'objet littéraire ni sur sa propre démarche productive. L'exercice suivant se proposait de faire réfléchir sur le destinateur, dans le modèle actanciel de Greimas.

\section{«Les écus du Ciel » («Die Sterntaler»)}

Les étudiants reçurent chacun un exemplaire du conte «Die Sterntaler» avec la consigne d'imaginer une suite pour la semaine suivante.

\section{Die Sterntaler}

Es war einmal ein kleines Mädchen, dem war Vater und Mutter gestorben, und es war so arm, daß es kein Kämmerchen mehr hatte, darin zu wohnen, und kein 
Bettchen mehr, darin zu schlafen, und endlich gar nichts mehr als die Kleider auf dem Leib und ein Stückchen Brot in der Hand, das ihm ein mitleidiges Herz geschenkt hatte. Es war aber gut und fromm. Und weil es so von aller Welt verlassen war, ging es im Vertrauen auf den lieben Gott hinaus ins Feld. Da begegnete ihm ein armer Mann, der sprach «ach, gib mir etwas zu essen, ich bin so hungrig». Es reichte ihm das ganze Stückchen Brot und sagte «Gott segne dirs», und ging weiter. Da kam ein Kind und jammerte und sprach «es friert so an meinem Kopfe, schenk mir etwas, womit ich ihn decken kann». Da tat es seine Mütze ab und gab sie ihm. Und als es noch eine Weile gegangen war, kam wieder ein Kind und hatte kein Leibchen an und fror: da gab es ihm seins; und noch weiter, da bat eins um ein Röcklein, das gab es auch von sich hin. Endlich gelangte es in einen Wald, und es war schon dunkel geworden, da kam noch eins und bat um ein Hemdlein, und das fromme Mädchen dachte «es ist dunkle Nacht, da sieht dich niemand, du kannst wohl dein Hemd weggeben», und zog das Hemd ab und gab es auch noch hin. Und wie es so stand und gar nichts hatte, fielen auf einmal die Sterne vom Himmel, und waren lauter harte blanke Taler: und ob es gleich sein Hemdlein weggegeben, so hatte es ein neues an, und das war vom allerfeinsten Linnen. Da sammelte es sich die Taler hinein und ward reich für sein Lebtag ${ }^{13}$.

Cet exercice gêna les étudiants dans un premier temps en raison du dépouillement systématique de la fillette, d'une exemplarité invraisemblable de nos jours et de l'intervention d'un deus ex machina qui sauve une situation narrative désespérée. Cependant, il donna lieu à une discussion utile sur le rôle du merveilleux dans le conte. Les suites envisagées furent très variées : l'héroïne fonde un orphelinat; un individu possède tout, mais ne trouve pas le bonheur ; la soudaine richesse change le caractère et la vie de la jeune fille; les gens de la ville trouvent cette fortune suspecte et dénoncent la fillette pour vol etc. Le décalage entre les époques respectives rendait impossible toute identification. La structure du conte: manque progressif jusqu'au dénuement total et intervention divine, permet de débusquer une instance morale, celle des adultes et des éducateurs, qui organise de tels récits. La confrontation avec un contre-exemple, le conte de la vieille femme dans «Woyzeck» de Büchner, précisa ce point.

Parmi les textes rédigés, citons celui-ci, d'une élégance astucieuse, que nous laisserons sans commentaire :

\section{Sterntaler (Eine Fortsetzung...)}

Das Mädchen ging mit der ganzen Knete in der Hand in die Stadt. Sie wollte sich zuerst ein paar andere Klamotten kaufen, denn sie fror erbärmlich in dem albernen Leinenfummel. Sie stand an der Kasse, hatte einen Berg von neuen Fetzen (Jacke wie Hose vom feinsten Stoff, einen Benetton Pulli und ein Krokodilhemdchen). Außerdem ein paar neue Jogging Treter, und auch sonst hatte sie die Taschen voll.

Da stand sie also an der Kasse und die olle, die dahinter saß, fragte sie, ob sie mit Visa Carte Bio oder gar mit American Express zahlen wollte. Natürlich hatte sie keinen von diesen Namen je gehört und schaute ganz bescheuert aus der Leinen wasche. Sie legte ein paar von ihren goldenen Talerchen auf den Tisch und wagte kaum etwas zu sagen. Da ging auch schon das Gelächter los: die Verkäuferin holte 
aus und schlug sich aufs Knie, die Kunden ringsherum bogen sich vor Lachen, keiner beobachtete das Mädchen, alle glotzten auf das Geld. Doch das Sterntalerfräulein war nicht blöd, packte ihre Jeans in die Tüte, sackte die Kohle ein und weg war sie. So hatte sie nun zwar wenigstens ein paar andere Kleider am Leib, aber sie merkte wohl, daß es gar nicht sehr einfach werden würde für sie in dieser Welt. Sie schlenderte durch die Gassen und schnupperte Großstadtluft. Ihr Weg führte sie an vielen teuren Läden vorbei und sie schaute alles sehr ungläubig an. Doch wie's halt so ist, mit den Sterntalerkindern: sie haben Glück! Denn kurz darauf fings an zu regnen. Es regnete zwar keine Kreditkarten oder sonst was außergewöhnliches, aber es regnete immerhin ganz schön. Sie duckte sich schnell an ein Schaufenster mit Vordach. Nachdem sie lange genug in dieses Sauwetter gestarrt hatte, schaute sie sich das Schaufenster an. Und was erblickten ihre müden Äuglein? Das ganze Fenster war voll von alten Münzen, gezackten, gelöcherten, silbrigen, goldenen, alten und neuen. Es lagen Bücher rum mit noch mehr Talern drauf. An der Scheibe stand in großen Buchstaben: Ankauf-Verkauf von Münzen jeder Art. Da brauchte selbst unser Sterntalerlein nicht lang zu überlegen. Sie ging rein, stapelte ihr ganzes Geld auf den Glastisch und lächelte dazu. Der Mann, der das sah, war von beidem dermaßen entzückt, daß er ihr dafür einen anständigen Stapel Geldscheine hinblätterte und sie ganz verliebt anstarrte. Sie nahm das Geld, lächelte nochmals höflich zurück und verließ erleichtert das Geschäft. Endlich war sie diese albernen Talerchen los und hatte harte DM in ihren zarten Händen!

\section{Les réécritures par des écrivains : Christa Wolf et Gunter Grass}

Jusqu'ici nous n'avons envisagé que des textes produits par des étudiants. Parmi les lecteurs qui passent à l'acte de la réécriture se trouve une catégorie particulière, celle des écrivains. Nous ne nous intéresserons pas, dans le cadre de cette étude, aux nombreux exemples de variations signalées dans des anthologies ${ }^{14}$, mais à l'insertion citationnelle et fragmentée de contes de Grimm dans des récits modernes, "Incident » de Christa Wolf $(1987)^{15}$ et « La Ratte » de Gunter Grass (1986) ${ }^{16}$.

\section{Christa Wolf}

Dans «Störfall», on trouve le passage suivant :

Was macht mein Kind

Was macht mein Reh

nun komm ich noch diesmal und dann nimmermehr.

[...] Immer wieder sind wir, verstoßen von der bösen Stiefmutter, in die Wildnis hinausgegangen, Hand in Hand, und die Brunnen, aus denen du trinken wolltest: Schwesterchen, mich dürstet. Wenn ich ein Brünnlein wüßte, ich ging und tränk einmal. Ich mein ich hört eins rauschen..., die Brunnen warnten uns mit ihren raunenden Stimmen, die ich dir übersetzte: Brüderchen, trink nicht. Sonst wirst du ein wildes Tier und wirst mich zerreißen. Da hat meine herzzerreißende Trauer immer 
angefangen, und oft und oft habe ich versucht, dir deinen Durst auszureden [...] Du wurdest ganz wild vor Durst und betteltest so lange um einen Schluck Wasser, bis ich dir erlauben mußte, unter Jammer und Wehklagen, aus dem letzten Brunnen zu trinken [...] und nun wurdest du [...] ein Reh [...] Ich legte dir [...] ein Band um den Hals und führte dich als Reh durch [...] ein[en dicht[en], undurchdringlichen] Wald, und wir fürchteten uns entsetzlich, bis wir [...] das Obdach gefunden hatten, in dem wir beide zusammen friedlich leben wollten, Brüderchen und

Schwesterchen [...] und ich habe dir, Brüderchen, oft und oft vorgehalten, daß ich, Schwesterchen mich doch auch bezähmen konnte; daß ich, obwohl so durstig wie $\mathrm{du}$, doch auch nicht unbedingt trinken mußte. Du aber mußtest unbedingt trinken, und du mußtest, trotz meiner Tränen und Bitten, in den Wald hinaus, als die Jagd vorbeikam, und so bist du auch es gewesen, der den fremden Königssohn bis an unseren Unterschlupf geführt hat, den ich doch gar nicht als Mann haben wollte, denn ich wollte nur dich, Brüderchen, und sei es als Reh [...]. Die falsche Schwester ward in den Wald geführt, wo sie die wilden Tiere zerrissen; die Hexe aber ward ins Feuer gelegt und mußte jammervoll verbrennen. Und wie sie zu Asche verbrannt war, verwandelte sich das Rehkälbchen und erhielt seine menschliche Gestalt wieder. Schwesterchen und Brüderchen aber lebten glücklich zusammen bis an ihr Ende ${ }^{17}$.

Quel est exactement le statut d'un tel fragment narratif? Le texte se lit comme une réduction plutôt fidèle (un "condensé » ou un "résumé») du conte "Frérot et sœurette $\aleph^{18}$. La trame, une partie des dialogues, les principaux épisodes sont sauvegardés. Le récit est présenté dans la perspective exclusive de la sœur. Il n'y a ni trahison, ni parodie, ni travesti. Une transformation par excision de cette ampleur reste-t-elle "purement formelle $\aleph^{19}$. A-t-elle une incidence thématique? Il faut bien s'interroger sur le statut, littéraire ou non, de ce fragment. La pratique du résumé est courante dans les encyclopédies, les manuels scolaires et répond généralement à des fins utilitaires. En aucun cas, note Genette, elle ne peut donner naissance à «de véritables œuvres ou textes littéraires $»^{20}$. Elle a de ce fait une fonction métalittéraire. En inversant alors la question, peut-on encore, dans ces conditions, parler ici de résumé $?^{21}$ Il semble que non, dans la mesure où font défaut les deux traits formels qui permettent de l'identifier comme tel, à savoir la narration au présent et à la troisième personne. Si nous avons une situation commentative, le contexte est totalement différent ${ }^{22}$. En effet, le commentaire ne porte pas sur l'hypotexte, le conte de Grimm, mais sur la signification symbolique du conte dans la biographie de la narratrice. Il a bien un statut littéraire et il est constitutif d'une stratégie discursive. Le conte est convoqué par l'instance narrative comme une réminiscence du passé. Les lieux et les objets familiers de l'enfance, la table, la cuisine, le robinet, prennent dans les jeux des deux enfants une signification symbolique et fonc-tionnent sur une scène imaginaire. Les scènes de l'enfance sont apparentées aux rêves: l'identification avec les personnages du conte relève, selon une expression de Henri James, d'une «crédulité consciemment cultivée $»^{23}$. L'écrivain recrée et revit artificieusement par l'écriture «la confusion supposée originelle entre le réel et l'imaginaire $»^{24}$. La narratrice (le Moi du récit) revît dans cet épisode la sortie de l'enfance et la fin des illusions pour la sœur (mais pas encore pour le frère, qui continue le «roman familial »"25). Elle bascule hors d'un univers où le temps est suspendu, où les angoisses se jouent et se dénient pour le monde des certitudes et de l'écoulement du temps, celui des adultes. Le décalage entre 
le «réel» du frère et celui de la sœur maintient encore deux univers désormais distincts et liés entre eux, chacun permettant de prendre la mesure de l'autre.

L'anamnèse est introduite par une séquence narrative: la narratrice attend avec angoisse des nouvelles de son frère qui souffre d'une tumeur au cerveau et subit une intervention chirurgicale longue et difficile. Comme dans le conte, le frère est en danger et la sœur ne peut conjurer totalement le destin. Elle connaît une tension intérieure telle qu'elle décide brusquement d'aller faire un tour de vélo dans la campagne. Le bruit d'un avion de chasse la terrifie «jusqu'au plus profond d'ellemême ", lui rappelle les bombardements de son enfance. Elle se réfugie dans la forêt. Le choc, l'odeur de la forêt, la similitude entre deux situations existentielles déclenchent le souvenir.

Christa Wolf installe ainsi une isotopie du dehors et du dedans: les agressions extérieures font écho aux agressions psychologiques, les chevreuils aperçus au loin rappellent celui d'un conte d'autrefois. Le passage dehors/dedans se double d'un passage présent/passé. La forêt, ce lieu tutélaire et mythique, constitue un véritable sanctuaire religieux où la narratrice se plaît à contempler les traces des rites et des civilisations d'un temps lointain. Elle représente un lieu priviligié où s'effectue le passage d'un univers à l'autre : l'auteur insiste sur le cercle, sur la lumière tamisée (le « filtre » de la conscience), sur l'orée, qui délimite deux espaces, l'un sacré ou magique et l'autre désenchanté.

L'insertion, sous forme condensée, du conte obéit à une stratégie discursive. Le souvenir d'enfance met en jeu deux aspects importants. D'une part, la répétition. Les enfants rejouent inlassablement les épreuves périlleuses de l'enfance et l'accession à une certaine maturité. D'autre part, la dénégation: il est possible de simuler des actions, de vivre des aventures qui mettent en jeu des relations mal maitrisées et angoissantes (en particulier incestueuses) tout en jouissant d'une impunité rassurante avec le bénéfice d'une juridiction d'exception. En outre, l'expérience (répétée) a montré que de telles situations traumatisantes avaient toujours une issue heureuse.

En conclusion, la «condensation » du conte de Grimm fonctionne dans le récit de Wolf à la fois par son contenu thématique, qui reste inchangé, et par les conditions même de son irruption dans la narration, mettant ainsi métaphoriquement en perspective la stratégie narrative de l'auteur.

\section{G. Grass}

Le roman de Grass, «La Ratte », relève d'une autre stratégie, que l'on pourrait définir comme une satire post-moderne. Il s'agit de mettre en garde les lecteurs des années quatre-vingt contre les dangers que court l'humanité et, par une « ironie militante $»^{26}$ en mêlant des souvenirs culturels du passé à des descriptions réalistes du présent et des anticipations vers un avenir apocalyptique.

Dans le chapitre II, l'auteur imagine que le Chancelier allemand décide de parcourir en limousine officielle, avec caravane et escorte, la « route allemande des contes de fées". En hâte, des ouvriers dressent sur un parking, situé au milieu d'une forêt malade, le décor qui accueillera son allocution devant les caméras et les journalistes ; 
An einem Stahlrohrgerüst ziehen Waldarbeiter [...] baumhohe Kulissen hoch, die mit gesundem Wald bemalt sind, etwa im Stil des Malers Moritz von Schwind: knorrige Eichen, dunkle Tannen, lichter Buchenbestand, der in unwegsamen Urwald übergeht. Es fehlen nicht Farn und Niederholz.

Auf hoher Leiter, die ein Spezialfahrzeug auszufahren verstand, malt ein Maler zusätzlich Singvögel - Buchfinken, das Rotkehlchen, etliche Singdrosseln, die Nachtigall - rasch wie gegen Stücklohn in die gemalten Baumkronen [...] Ruckzuck räumen die Waldarbeiter die Szene. Das Polizeivorauskommando verteilt sich und sichert das Gelände. Hinter den Kulissen schaltet ein Tonmeister ein Tonbandgerät ein. Wir hören reichgemischt Vogelstimmen, unter ihnen die frischgemalten Buchfinken, Rotkehlchen, Singdrosseln, aber auch einen Pirol und mehrere Waldtauben $[. . .]^{27}$

Grass construit avec une énumération luxuriante un effet de réel, l'univers référentiel, d'un épisode de son roman. Mais il a pris soin de préciser le caractère factice de cette construction littéraire dont la responsabilité morale incombe... aux politiciens et aux "spécialistes" de la communication moderne. Le Chancelier s'adresse à ses concitoyens sur l'écran de télévision dans un décor qui reconstitue l'univers perdu des contes de Grimm. Il parle avec une aisance acquise et tient sur les problèmes de son pays des propos lénifiants et rassurants: la société et l'environnement sont menacés par des $«$ nuisances $»^{28}$. Grass oppose discrètement, dans ce passage, la parole du romancier et celle, pourtant «imagée» (!), des hommes politiques. Il enchâsse l'allocution du Chancelier, réduite à un résumé singulièrement laconique, dans une séquence où le romancier décrit en détail la construction d'un décor en trompe-l'œil. L'image diffusée par les média est dénoncée comme (dis)simulation : la forêt malade du récit est masquée par une représentation de la forêt saine d'autrefois, qui est de surcroît une copie des illustrations populaires du XIX ${ }^{e}$ siècle $^{29}$. À cette première déconstruction du discours concurrent sur les dangers du monde moderne succèdent une seconde, puis une troisième. Les enfants du Chancelier "font irruption dans le cadre", ils se révoltent et sèment la pagaille. Le fils saute sur l'estrade et dénonce publiquement les ravages de l'industrie sur la forêt. Puis la fille entre en action. Armée d'un couteau de chasse, qu'elle vole au garde forestier, elle tranche les cordes qui retiennent le décor :

In Zeitlupe fallen die Kulissen in sich zusammen. Kein gemaltes Vöglein fliegt, sich rettend, davon. Kein Reh Hase Igel flüchtet. Nicht nur das Stahlrohrgerüst, der tote Wald steht unübersehbar. Jetzt stellt die Tochter das Tonband mit den Vogelstimmen ab. Stille. Dürres Geäst knackt, bricht. Mit dem Schwindel fliegen Krähen auf. Angst geht um, unumschrieben, der $\operatorname{Tod}^{30}$.

La mort qui rode, et qui surgit lorsque le truquage s'effondre, est une mort baroque. L'auteur se fait prophète et son récit illustre, comme une répétition générale, le sort qui attend les hommes. À ce moment précis, Grass accomplit l'identification des deux enfants du Chancelier avec Jeannot et Margot. Ils déshabillent les comédiens qui jouaient leur personnage et s'enfuient dans la forêt «comme Jeannot et Margot » ${ }^{31}$. On les retrouve soixante pages plus loin alors qu'ils traversent en courant une forêt morte, longeant des décharges sauvages, des dépôts toxiques et des bases militaires ${ }^{32}$. Remontant le temps, ils s'enfoncent dans une végétation de plus en plus verdoyante et se retrouvent... dans la forêt germanique des contes de fées, que l'auteur décrit avec un 
luxe de détails et le plaisir évident du conteur. Les deux enfants avancent "sans effort " et «pas désespérés le moins du monde » (p. 113). Le narrateur convoque au fil du récit tous les personnages des contes de Grimm qui accueillent les fugitifs : les sept cygnes, le loup, la grenouille, mais aussi Blanche-Neige et la méchante belle-mère, la Belle au Bois Dormant et son prince charmant, le Chaperon Rouge, Riibezahl, Rapunzel, la jeune fille sans mains, Jorinde et Joringel etc. Grass glisse malicieusement quelques anachronismes, comme ce distributeur automatique des années cinquante, des phantasmes de l'enfance, comme par exemple les énormes tétons de la Dame. Si cette dernière est identifiée comme la « sorcière » de tous les contes, elle est décrite comme «ni jeune ni vieille», «à la fois laide et belle ». Personnage générique, elle porte la marque des commentaires du $\mathrm{xx}^{\mathrm{e}}$ siècle sur le conte et sa structure (Propp, mais aussi Benjamin, Bloch, Bettelheim ${ }^{33}$ ). Décrite avec des bigoudis et un peignoir, portant autour du cou une chaîne où sont enfilées des oreilles séchées, et agitant frénétiquement la crécelle des fous, elle vient directement de la tradition grotesque.

51 On retrouve le programme narratif du conte initial (la fuite, la forêt, la cabane de la sorcière, etc.). Mais Grass procède à une relecture des frères Grimm. Les personnages ne connaissent plus la peur. Les "monstres" des contes sont des connaissances familières. Chacun présente aux autres son histoire et ses reliques. L'idéalisation du passé a lieu dans un univers baroque : les personnages exhibent leurs mutilations avec fierté, le Chaperon Rouge lance des insolences à sa grand-mère, en galant homme Rumpelstilzchen porte un verre de jus de baies aux lèvres de la fille sans mains etc. Le narrateur suggère que ces êtres qui hantaient l'imaginaire de notre enfance sont plus humains et plus inoffensifs que les hommes $d u x^{e}$ siècle. Les actions relatées dans les livres d'image sont répétées, exposées, résumées, et perdent ainsi leur caractère effroyable. Nous déambulons dans un univers idyllique et confraternel qui représente dans le roman une enclave protégée contre les dégradations de l'histoire. Grass exprime, sur le mode du paradoxe et de la nostalgie, son angoisse devant les ravages du progrès. Il reprend à son compte l'image de «l'ange de l'histoire » de Benjamin, une figure qui représente le progrès : son visage est tourné non vers l'avenir, mais vers le passé. "Il ne voit qu'une seule et unique catastrophe qui ne cesse d'amonceler ruines sur ruines et les jette à ses pieds $»^{34}$. Dans le même temps, les impertinences de l'auteur (ses ajouts "grotesques") sont autant d'indices douloureux d'une dégradation du patrimoine culturel. La nature, écrit Grass, est malade de l'homme :

Weil der Wald

an den Menschen stirbt,

fliehen die Märchen,

weiß die Spindel nicht,

wen sie stechen soll,

wissen des Mädchens Hände nicht,

die der Vater ihm abgehackt,

keinen einzigen Baum zu fassen,

bleibt der dritte Wunsch ungesagt $[\ldots]^{35}$.

52 Dans un monde «moderne » menacé de " décomposition »" procède à la dislocation du monde des contes de fées. À la mise en échec de l'humanité, il répond - en tant qu'écrivain - par la mise en échec d'une logique qui structurait notre imaginaire. Mais il le fait sur le mode jubilatoire qui permet le dépassement créatif du désespoir. 
53 gratitude, Grass réintroduit dans l'univers romanesque moderne tous les exclus, les laissés-pour-compte, les minorités opprimées, les boucs émissaires qui peuplent les poubelles de l'histoire. Les rats représentent tout ce que l'homme rejette hors de la sphère de la culture et de la technologie, ils prolifèrent dans les déchets de la civilisation et survivront, seuls, à son anéantissement. Au lyrisme du progrès succède étrangement la prophétie d'une décomposition générale. Et le dialogue du narrateur avec la ratte, qui constitue le roman, dénonce «la langue des illusionnistes» («die Täuschersprache» $)^{37}$ de ceux qui s'agitent sur la scène pour donner le change. Ce faisant, Gunter Grass règle des comptes avec ses contemporains en inventant un conte post-moderne.

\section{NOTES}

1. - Nos remerciements vont aux collègues de la PH de Ludwigsburg, en particulier H. Melenk, aujourd'hui président de la PH, et Karlheinz Fingerhut, professeur de littérature allemande, qui a eu l'initiative de ce séminaire. Nous renvoyons aux études de K. Fingerhut sur ce thème, en particulier : «Umerzählen von Texten - Eine Möglichkeit, Textanalyse und Textproduktion zu verbinden», in Wissenschaft und Unterricht, 1980, n³, p. 176-198; «Umerzählen. Texte und Materialien zum Literaturunterricht», Diesterweg, Francfort/M, 1982.

2. - Propp, « Morphologie du conte », Seuil, Paris, 1970. Claude Brémond, «Logique du récit », Seuil, Paris, 1973.

3. - En particulier « Neigeblanche et Roserouge » («Schneeweißchen und Rosenrot», «Kinder-und Hausmärchen, gesammelt durch die Brüder Grimm», Winkler-Verlag, Munich, 1963, p. 674-675), « Blanche-Neige » («Schneewittchen») (le thème du miroir, Grimm, p. 297-308).

4. - Nous laissons de côté l'interprétation d'Erich Fromm qui décèle dans ce conte une haine farouche et triomphante de l'homme et de la sexualité (ici inversée en attirance contrôlée). E. Fromm : «Märchen, Mythen, Träume», Rowohlt, 1987, p. 157-160.

5. - Nous signalons en italique les ajouts de l'auteur. Thaddäus Troll (1963, réécrit en 1980), in Hans Ritz, «Die Geschichte von Rotkäppchen. Ursprünge, Analysen, Parodien eines Märchens», Heyne, Munich, 1986, p. 120.

6. - Grimm, op. cit., p. 112.

7. - Marthe Robert, préface aux «Contes de Grimm », Gallimard, Paris, 1976, réédition Folio (1990), p. 14.

8. - Ibid., p. 12.

9. - Ibid., p. 14-15. Il convient cependant de noter que la mission de la «fée " chez les frères Grimm est d'assister l'enfant ou l'adolescent à s'intégrer dans un ordre social immuable, tandis que la version moderne propose de changer les valeurs sociales.

10. - «Le "royaume" du conte en effet, écrit Marthe Robert, n'est pas autre chose que l'univers familial bien clos et bien délimité où se joue le drame premier de l'homme ». Les enfants y croient « dans la mesure même où ils y trouvent [...] une image identifiable d'eux-mêmes, de leur famille, de leurs parents ", op. cit., p. 18. 
11. - Claude Brémond, «La logique des possibles narratifs », Communication, n8, 1966, p. 60-76. Sur cette question, on lira l'étude remarquable d'Els Andringa, «Lese-voraussetzungen und die Rezeption literarischer Figuren», in Diskussion Deutsch, 1988/12, p. 622-644.

12. - C. Brémond, op. cit., p. 63, « Logique du récit », Seuil, Paris, 1973, p. 71.

13. - Grimm, op. cit., p. 666-668.

14. - Signalons cependant, outre Fingerhut (1982), p. 9-41, Hans Ritz, op. cit.; Wolf-gang Mieder (éd.), «Grimms Märchen modem», Reclam, Stuttgart, 1979 ; Jack Zipes, «Rotkäppchens Lust und Leid. Biographie eines europäischen Märchens», Ullstein, 1985, Rosemarie Lauer/Wolfgang Mieder, «Kein Hansel ohne Gretel», Eichborn Verlag, Francfort/M, 1988.

15. - C. Wolf, «Störfall. Nachrichten eines Tages», Luchterhand, Darmstadt, 1987. Traduction française de Ghislain Riccardi, «Incident. Nouvelles d'une journée », Alinéa, Aix-en-Provence, 1989.

16. - G. Grass, «Die Rättin», Luchterhand, 1986, nous citons l'éd. Rowohlt de 1988, trad. fr. de Jean Amsler, « La ratte », Seuil, Paris, 1987.

17. - «Störfall», p. 80-82.

18. - «Brüderchen und Schwesterchen», Grimm, p. 91-103. La réduction s'évalue à $10 \%$ du volume de l'hypotexte.

19. - Gérard Genette, « Palimpsestes », Seuil, Paris, 1982, p. 263.

20. - Ibid., p. 280-283.

21. - Nous écartons l'hypothèse du digest qui « raconte à sa manière nécessairement plus brève (c'est sa seule contrainte) la même histoire que le récit » (Genette, p. 283-284) mais se présente comme un récit parfaitement autonome, sans référence à l'hypotexte, alors que Wolf insère à plusieurs reprises des séquences métadiscursives qui mettent la source littéraire en perspective. 22. - Le résumé de roman, écrit justement Weinrich, «sert d'appui au commentaire de l'œuvre littéraire » («Dient gewöhnlich der Besprechung eines literarischen Werkes als Grundlage»). Il «figure dans des guides de lecture en forme de dictionnaire; l'ordre alphabétique ou chronologique y fait déjà contexte ». Il se présente toujours en situation commentative («als Teil einer Situation des Besprechens»). Harald Weinrich, «Tempus», Kohlhammer, Stuttgart, 1971, p. 44. Trad. fr. « Le Temps », Seuil, Paris, 1973, p. 41-42.

23. - Cité par O. Manonni, « Clefs pour l'imaginaire ou l'Autre Scène », Seuil, Paris, 1969, p. 167.

24. - O. Manonni, op. cit., p. 168.

25. - «Aber eines Nachts fragtest du mich, ob ich nicht etwa die falsche Schwester sei [...] da mußte ich zur stärksten Formel greifen [...]: tot umfallen will ich, wenn ich nicht die richtige Schwester bin. Darauf schwiegst du ein Weilchen, Brüderchen, und fragtest dann vorsichtig: Bist du tot umgefallen?, und ich sagte, Trauer, Trauer im Herzen: Nein. - Da war nun bewiesen, was eines Beweises nie hätte bedürfen solle», op. cit., p. 82 .

26. - Northrop Frye, « Anatomie de la critique », Gallimard, Paris, 1969, p. 272.

27. - «Die Rättin», p. 46.

28. - «Jetzt spricht [...] der Kanzler, indem er geübt vom Blatt liest. In Bildern beschwört er eine heile Welt, die von Ungemach bedroht ist», «Die Rättin», p. 48.

29. - Moritz von Schwind (1804-1871) fut avec Franz Pocci (1807-1886), Otto Speckter (1807-1871) et d'autres, l'un des premiers illustrateurs, parmi les plus connus, des contes de Grimm.

30. - «Die Rättin», p. 49.

31. - «Sohn und Tochter des Kanzlers reißen den kostümierten Märchenfiguren Hansel und Gretel die Mütze, das Häubchen ab, werfen ihren Walkman weg, schneiden Vater und Mutter und obendrein dem Fernsehen Grimassen und laufen aus freien Stücken, der Grimmschen Märchenfassung spottend, als Hansel und Gretel in den Wald» (49-50).

32. - «Die Rättin», p. 112.

33. - Cf. Fritz, op. cit., p. 7-19 et les indications bibliographiques, p. 154. 
34. - W. Benjamin, «Geschichtsphilosophische Thesen», in "Zur Kritik der Gewalt und andere Aufsätze», Suhrkamp, 1964, p. 84-85, trad. fr. M. de Gandillac, « Poésie et Révolution », Denoël, Paris, 1971, p. 282.

35. - «Die Rättin», p. 43-44.

36. - Nous renvoyons à l'anecdote signalée par Welsch : un jour un passant distrait lut sur les panneaux de sa ville " Munich se décompose » au lieu du slogan promotionnel «Munich se modernise ». Le renversement de sens est le résultat d'un jeu de mots qui substitue le verbe «modem» (moisir) à l'adjectif «modem» (moderne). Wolfgang Welsch, «Modernité et postmodernité », Les Cahiers de Philosophie, Lille, 1988, n6, p. 22.

37. - «Die Rättin», p. 66.

\section{RÉSUMÉS}

Les contes de Grimm ont été réécrits au cours d'un séminaire par des étudiants de la PH de Ludwigsburg. En s'appuyant sur plusieurs exemples, l'auteur étudie les modifications thématiques et leurs significations. Les travaux de Bremond sur le récit permettent de mettre en évidence, et à l'épreuve, la structure fondamentale du conte. L'auteur s'intéresse aux formes et aux enjeux (narratifs, individuels et pédagogiques) d'une actualisation du vocabulaire, et des variations sur le «procès de socialisation» proposé par le conte, ainsi qu'à l'investissement personnel du nouvel « auteur ».

Une seconde partie examine le statut discursif des contes de Grimm insérés dans deux romans contemporains : «Incident» (1987) de Christa Wolf, et «La Ratte» (1986) de Günter Grass. L'évocation, en partie citationnelle et en partie métalinguistique, de «Frérot et sœurette » dans « Incident » plonge le lecteur dans des situations angoissantes de l'enfance pour montrer qu'elles sont surmontables. Grass, dans un récit post-moderne, oppose au monde contemporain malade la déconstruction nostalgique et grotesque de l'univers des contes allemands.

Grimms Märchen wurden im Rahmen eines Seminars von Studenten der PH Ludwigsburg umgeschrieben. Es wird unter Heranziehung mehrerer Beispiele untersucht, welche inhaltliche Veränderungen mit welcher Absicht vorgenommen wurden. Unter Berufung auf die Arbeiten von Bremond über die Narrativik wird die Grundstruktur des Märchens erschlossen und auf die Probe gestellt. Dabei wird differenziert, inwieweit und wozu der Wortschatz aktualisiert wurde, welche Variationen zum Sozialisationsprozeß des Märchens angeboten werden, und inwieweit sich der neue «Autor» variierend in die (um)erzählte Welt hineinprojiziert.

In einem zweiten Teil, wird nach dem kommentierenden Statut eingeschobener Märchen in Christa Wolfs «Störfall» (1987) und Günter Grass' «Die Rättin» (1986) gefragt. In «Störfall» versetzt uns die teils zitathaft und teils metasprachlich vermittelte Erinnerung an «Brüderchen und Schwesterchen» in die Kindheit, wo angst auflösende Situationen als überwundbar nacherlebt werden. Grass stellt der kranken Gegenwart eine groteske und sehnsüchtige Dekonstruktion der deutschen Märchenwelt in einer postmodernen Erzählung gegenüber. 